\title{
Application of Numerical Methods for Research of Construction Design of Fastener Fractures
}

\author{
Leonid Kolomiets*, Aleksej Aniskin, Victor Orobey, Alexander Daschenko, Alexander Lymarenko, Kostiantyn Boriak
}

\begin{abstract}
The work uses modern numerical methods of mechanics of a deformable solid to analyze the stress-strain state of orthopedic structures in order to improve them and improve the quality of treatment of patients. Among the many numerical methods, the attention of the authors was drawn to the finite element method and to the numerical and analytical method of boundary elements in the author's edition. Settlement models of both metal apparatuses and parts of a person's arm with a fracture are constructed. The stress - strain state in various zones of the biomechanical system was calculated. It is shown that the numerical-analytical method of boundary elements allows obtaining more accurate results with fewer equations in the resolution system. It is noted that in the case of the considered biomechanical systems, its elements undergo tension - compression, shear, torsion, and bending. To consider them, solutions are presented for Cauchy problems, which are included in the general system of resolving equations of the MGE. It is shown that, unlike the FEM, the MGE simplifies the algorithm for calculating biomechanical systems. Comparison with the FEM results shows their good agreement, which proves the reliability of the calculation results of both methods.
\end{abstract}

Keywords: boundary element method; finite element method; orthopedic constructions; stress-strain state

\section{INTRODUCTION}

One of the ways to investigate various designs in medicine is a computer simulation or computer experiment based on numerical methods (finite difference method, boundary element method, finite element method, and others) $[1,2]$.

The capabilities of the numerical method depend on task and applied software package.

Numerical analysis allows you to calculate various design options of the studied object and take into account various combinations of loads.

The relevance of the research topic, the problems with the treatment of patients and of the forearm bones diaphysis fractures (compact bone substance), is determined by the insufficient perfection of the devices and the appropriate treatment methods $[3,4]$.

\section{ANALYSIS OF RECENT RESEARCHES AND PUBLICATIONS}

From literary sources it is known that forearm bones fractures account for $5-15 \%$ of cases from all fractures of limb segments.

Ilizarov apparatus was the most widely used for the treatment of patients with injuries of limb segments [2]. The use of this apparatus gives a significant percentage of complications due to a decrease in the stability of the spoke fixation and the bulkiness of the external structure. This was the reason for the search by many scientists and researchers in the field of traumatology for alternative configurations of compression-distraction apparatuses (various designs of external supports, node connections, types of fixation).

Research overview $[5,6]$ shows that rod apparatus from the alternative devices for transosseous fixation have obvious advantages. Some restrictions of their use are associated with existing problems in osteosynthesis techniques depending on the type and pattern of the diaphyseal fracture of the bones (in our case the forearm).

During the work with literary sources [1-8] data gaps in comparative calculations of external structures for the treatment of forearm fractures by numerical methods became apparent.

\section{THE PURPOSE OF WORK}

The purpose of the work is the justification for the external fracture fixation design and methods for determining the deformation of geometric dimensions.

Calculation of the stress-strain state of cylindrical shells of BEM and FEM (ANSYS) [9-12] and comparison of their results (MATLAB).

\section{MAIN PURPOSE OF THE ARTICLE}

The basis of the research of this design is the purpose to design a fracture treatment device of the middle part of the forearm with damage to the diaphysis of the tubular bones by developing an alternative design in which the reposition of fragments that have come out of the correct state is achieved by increasing the degrees of mobility of the repair nodes. Thanks to this design it becomes possible to ensure the correct position of displaced bone fragments without gypsum immobilization and most importantly full mobility of the forearm is maintained without creating inconvenience in the patient due to compactness.

The problem is solved thanks to the external support in the construction in the form of a beam on which rod receivers with screw studs are located fixing screw rods that are successively passed through parts of intact bones to ensure their rigid connection [2].

The data for building models were obtained based on the transverse scans of the bones of the human forearm carried out on a TOSHIBA Asteion Super 4 multislice helical 
computer scanner. Further 3D model was created that adequately reflects the geometry of the bones of the forearm.

We conducted a computer simulation of fixation methods in order to determine the stiffness of fixation of fractures of the forearm bones with an apparatus for transosseous osteosynthesis with their specific layout and in the range of possible functional loads, identifying the optimal arrangement of the apparatus, determination of treatment tactics depending on the type of fracture.

From the point of view of mechanics, such systems are spatial structures from a set of thick-walled annular and rectangular plates with holes, classic rods, flexible rods (spokes) in combination with various types of joints.

The simplified analytical methods of calculation are often used among the methods of mechanics in engineering practice and scientific research. The design scheme of the investigated object is simplified both by the design of the elements of the spatial system, the nature of the relationship between each other and from the point of view of representing external loads. The widespread use of modern computer technology in research projects required a revision of existing methods. If so far, the main attention has been paid to the creation of calculation methods based on a number of simplifying assumptions and methods of artificially facilitating the calculation, then at present we can only talk about the development of mathematical models that are subject to the most efficient implementation on a computer. We mean here by effective such models that most fully reflect the real geometry and behavior of the structure require simple software and the least amount of machine time.

The most convenient for solving problems of mechanics both in medical research and in other fields of science and technology turned out to be the methods of the discrete theory of linear spaces: matrix calculus, the potential method, the boundary element method and of course the finite element method (FEM).

To solve the FEM problem it is necessary to present the entire construction in the form of a set of finite elements: rods, triangular and quadrangular plates, tetrahedrons and parallelepipeds connected to each other in nodes.

The initial stage in the finite element analysis of any constructions including orthopedic devices is the construction of a geometric and finite element model.

The geometric model represents the shape and dimensions of the construction and the finite element model contains complete information about the location of nodes and finite elements as well as the relationship between individual nodes and elements.

Theoretically, it is possible to carry out finite element analysis without using a geometric model but in this case, it is necessary to manually set the coordinates of all nodes and build the finite elements of the model, which is almost impossible for complex constructions.

Using a geometric model this drawback is absent as it is possible to automatically obtain a finite element mesh of a structure based on its geometric model in modern programs of finite element analysis [9, 12]. Therefore, such an approach to modeling orthopedic systems is preferable.

Elements of the core system of fracture treatment devices can be modeled using the following types of finite elements:

- beam and core elements

- plate elements

- volumetric elements.

The geometric model must comply with the type of element that will be used in the finite element breakdown. If the discretization of the structure will be carried out by beam and rod elements, then the geometric model should consist of lines, for plate elements - from surfaces, for volume elements - from volume bodies.

The paper considers models with rod and volume finite elements, because they ensure compliance with the geometry of the model of a real construction (Fig. 1).

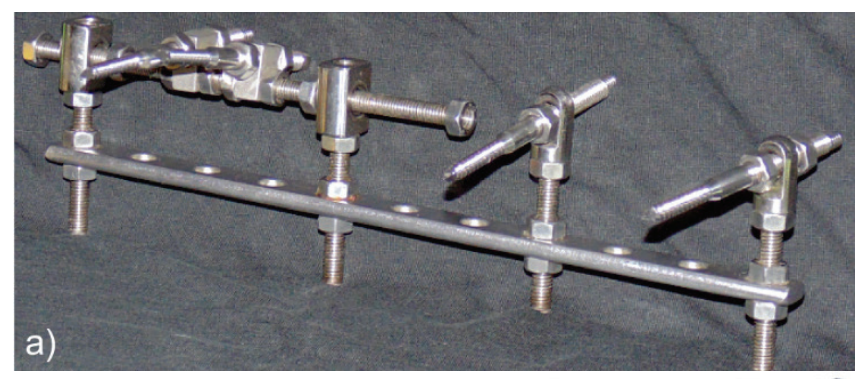

b)

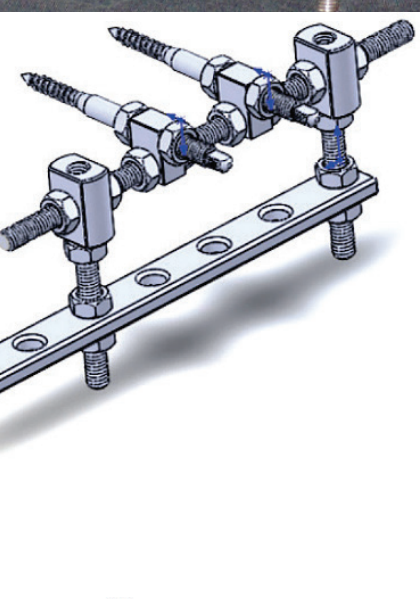

c)

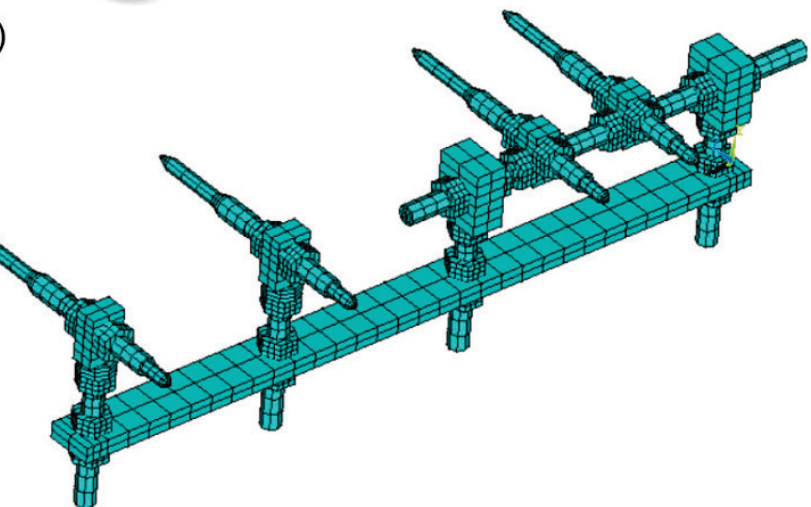

Figure 1 Real (a) and computer models of volumetric finite elements (b) and rod finite elements (c)

An approximation of model from a rod finite elements was done the BEAM188 element from the library of elements of the ANSYS program. The element is intended to solve spatial problems. Element properties are defined by describing the cross-sectional characteristics, material 
properties (modulus of elasticity and Poisson's ratio) and elastic base.

This finite element has two nodes with six degrees of freedom and each of them: displacements in the directions of the $x, y, z$ axes of the nodal coordinate system and rotation angles around these axes.

To display geometric features original cross sections of the structural elements of the clamps were formed and a special function of the ANSYS - Taper Section program was used (we have created and recorded in the database the 37 cross-section programs).

The finite element partition of the model with volume elements is performed by the Solid45 element. The element is classified as follows: a volumetric (3D) element is used in solving problems of the mechanics of a deformable solid and is determined by eight nodes, each of which has three degrees of freedom - linear displacements in the directions of the $x$, $y, z$ axes of the nodal coordinate system.

In both cases the fixing conditions should be chosen so that they prevent the movement of the structure but do not affect the deformation.

The geometric and mechanical characteristics of the studied devices were determined by the known mechanics dependencies of a deformable solid, experimental data and reference books.

The elastic modulus of the human bone was accepted equal $E_{\mathrm{K}}=2 \times 10^{4} \mathrm{MPa}$, modulus of elasticity of steel $E_{\mathrm{cm}}=$ $2.1 \times 10^{5} \mathrm{MPa}$, modulus of elasticity of titanium $E_{\mathrm{m}}=1.2 \times 10^{5}$ MPa (Fig. 1).

As a result of calculations for each of the options the parameters of the stress-strain state that were of interest to us for the analysis were determined:

$\sigma_{\text {equiv }}$ - equivalent stresses according to the Huber-Mises hypothesis (IV theory of strength)

USUM - equivalent displacement values.

\subsection{Application of the Numerical-Analytical Version of the Boundary Element Method}

According to the accepted design scheme (Fig. 2) it may be considered that the orthopedic structure and the forearm bones form as a first approximation a spatial rod system. This system uses both hingedly connection of the rods and hard jamming of structural elements. These boundary conditions can be accurately taken into account if we apply a numericalanalytical version of the boundary element method (BEM). In general, the elements of a given biomechanical system will experience 4 types of resistance: tension-compression, shear, torsion, and bending. In this case, at the boundary points $(x=$ 0 and $x=1$ ), kinematic and static boundary parameters arise:

a) Tensile compression:

$U(0), U(l)$ - movement along the axis of the rods, $N(0), N(l)$ - normal forces;

b) Shift:

$V_{\mathrm{c}}(0), V_{\mathrm{c}}(l)-$ cross movements

$Q(0), Q(l)-$ cross forces. c) Torsion of solid section rods:

$\theta(0), \theta(l)$ - twisting angles of boundary points

$M_{\mathrm{kp}}(0), M_{\mathrm{kp}}(l)-$ torques.

d) Bend:

$V(0), V(l)$ - cross movements;

$\varphi(0), \varphi(l)$ - turning angles of the sections at the boundary points

$Q(0), Q(l)$ - cross forces

$M(0), M(l)$ - bending moments.

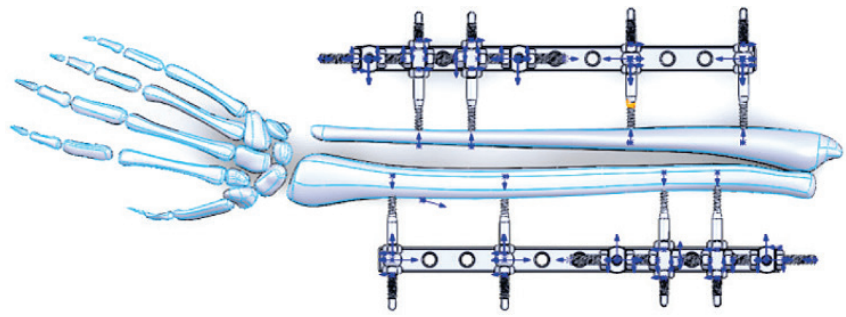

Figure 2 The design scheme of the object of study

In the formation of the permissible equations of the BEM, we will use the left-handed coordinate system and the following sign rules:

- Linear movements of boundary points are considered positive if they coincide with the positive directions of the axes $0 x, 0 y, 0 z$.

- Turning angles of rotation of the sections at the boundary points are considered positive if they are clockwise when viewed from the direction of the positive axes $0 x, 0 y, 0 z$. As an example Fig. 3 shows the positive directions of the boundary parameters during bending.
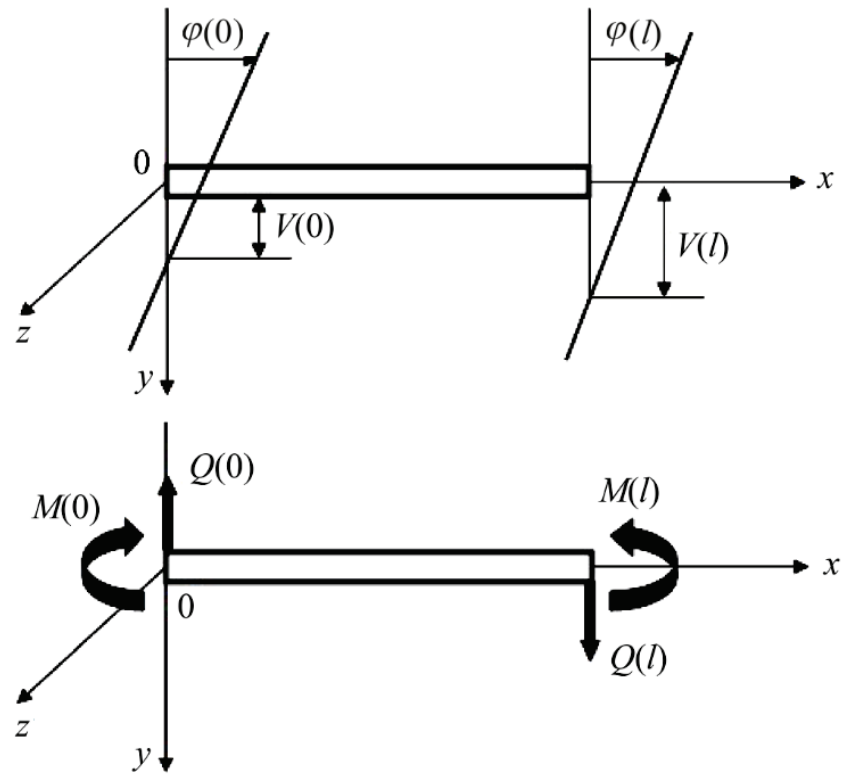

Figure 3 Positive directions of the boundary parameters during bending

In the case of deformation of the core system, the nodes receive certain linear and angular displacements, and the kinematic parameters in the nodes will be related by the 
equations of compatibility of displacements. Accordingly, the static parameters in the nodes will be related by equilibrium equations. These considerations allow us to form the resolving equations of the BEM of any core system. The solution of this system determines the stress-strain state of all structural elements.

Let us imagine solutions to Cauchy problems for the indicated types of resistances:

a) Tensile compression

\begin{tabular}{|c|}
\hline$E A U(x)$ \\
\hline$N(x)$ \\
\hline
\end{tabular} \mid \begin{tabular}{|c|c|}
\hline 1 & $x E A U(0)$ \\
\hline & $1 N(0)$
\end{tabular}$-\int_{0}^{x} \begin{gathered}(x-\xi) q x(\xi) \\
q x(\xi) \\
\mathrm{d} \xi\end{gathered}$

\begin{tabular}{|c|c|c|c|c|c|}
\hline$E I V(x)$ & 1 & $x$ & $\frac{-x^{2}}{2}$ & $\frac{-x^{3}}{6}$ \\
\hline$E I \varphi(x)$ & $=$ & 1 & $-x$ & $\frac{-x^{2}}{2}$ \\
\hline$M(x)$ \\
\hline$Q(x)$ \\
\hline
\end{tabular}

d) Bend

b) Shift

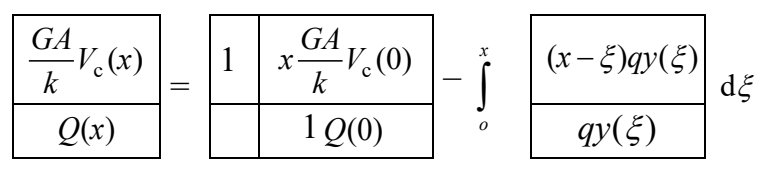

c) Torsion of solid section rods

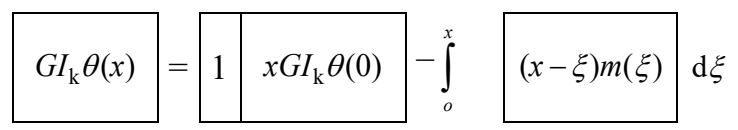

Using these solutions and the BEM algorithm it is possible to formulate the resolving BEM equation, find the unknown initial and final parameters of all elements of the biomechanical system and determine its stress-strain state.

\subsection{Solving Boundary Value Problems of Biomechanical Systems BEM}

If the rods of the biomechanical system are connected into a single structure then for such system it is possible to apply matrix forms of solutions to Cauchy problems (1)-(4). In this case, the matrix equation of the whole structure will take the form

$$
\boldsymbol{Y}(x)=\boldsymbol{A}(x) \boldsymbol{X}(0)+\boldsymbol{B}(0)+\boldsymbol{B}(x)
$$

where: $\boldsymbol{Y}(x)$ - vector of deformation parameters in the current section; $\boldsymbol{A}(x)$ - quasi-diagonal matrix of fundamental functions of all kinds of resistances; $\boldsymbol{X}(0)$ - vector of initial parameters; $\boldsymbol{B}(x)$ - load matrix.

At the boundary value of the parameters $x=l_{i}$ (for all rods) there arises the possibility of an elementary transformation of Eq. (5) into the resolving equation of the BEM boundary value problem according to the scheme

$$
\boldsymbol{Y}(l)=\boldsymbol{A}(l) \boldsymbol{X}(0)+\boldsymbol{B}(l) \rightarrow \boldsymbol{A}(l) \boldsymbol{X}(0)-\boldsymbol{Y}(l)=-\boldsymbol{B}(l)
$$

As stated above, coupling equation between the boundary parameters arise in the nodes of the rod system. This fact allows us to transfer all the finite vector options $\boldsymbol{Y}(l)$ on vector $\boldsymbol{X}(0)$. In this case, the quasi-diagonal matrix $\boldsymbol{A}(l)$ will be reset to separate columns and compensating nonzero elements are introduced into them as initial data; as a rule, this is $(-1)$. Thus, a system of resolving equations is formed with respect to the initial and final parameters of the entire structure of the form

$$
\boldsymbol{A}_{*}(l) \cdot \boldsymbol{X}_{*}(0, l)=-\boldsymbol{B}(l)
$$

Note that the load $\boldsymbol{B}(l)$ matrix is not involved in these transformations, which favorably distinguishes the BEM algorithm from the finite element method, where it is necessary to reduce the given load to the equivalent nodal the matrix transformation $\boldsymbol{A}_{*}(l)$. The following stages are stand out here:

- a quasi-diagonal matrix is formed $A(l)$; its filling with blocks of boundary values of orthonormalized fundamental functions is performed according to the program using cycle operators;

- matrix columns are reset to zero $A(l)$, whose numbers are equal to the vector zero line numbers $\boldsymbol{X}(0)$. Zero initial parameters of the rods are the source data and the numbers of zero lines of the vector $\boldsymbol{X}(0)$ are determined in the process of its formation. We denote the matrix nullable in separate columns $\boldsymbol{A}_{0}(l)$.

- compensating elements are determined that allow to transfer all the finite parameters of the vector $\boldsymbol{Y}(l)$ to the vector $\boldsymbol{X}_{*}(0, l)$. The compensating elements can be reduced to an auxiliary matrix $\boldsymbol{C}$ for clarity of the algorithm. This matrix characterizes the design topology and is invariant with respect to the type of calculation, i.e. can be used without any changes in tasks of statics, dynamics and stability. The coefficient matrix of the system of Eq. (7) is obtained by simple summation

$$
\boldsymbol{A}_{*}(l)=\boldsymbol{A}_{0}(l)+\boldsymbol{C}
$$
load. The most complex equation formation operation (7) is 
A system of resolving Eq. (7) is compiled for the design scheme according to Fig. 2. The results of determination of the stress-strain state of the construction according to the BEM algorithm are presented in Tab. 1. From the data Tab. 1 it follows that the results of the BEM and FEM methods are good accord with each other, which proves the reliability of these calculations.

Table 1 The maximum values of the stress-strain state parameters

\begin{tabular}{|l|c|c|}
\hline \multicolumn{1}{|c|}{ SSS parameters } & Fixator (FEM) & Fixator (BEM) \\
\hline$U S U M(\mathrm{~mm})$ Elbow joint area & 0,00197 & 0,00186 \\
\hline$U S U M(\mathrm{~mm})$ Fracture area & 0,0089 & 0,0075 \\
\hline$U S U M(\mathrm{~mm})$ Wrist area & 0,0273 & 0,0218 \\
\hline${ }_{\max } \sigma_{\text {equiv }}(\mathrm{MPa})$ & 16,098 & 21,783 \\
\hline
\end{tabular}

The maximum values of the specified stress-strain state parameters for all calculation options are summarized in Tab. 1.

\section{RESULTS}

The results of a numerical experiment allowed drawing some conclusions:

Relative deformation of the rod apparatus:

Model from FE: $\varepsilon_{\mathrm{c}}=\frac{\Delta l}{l}=\frac{0.0273}{235}=1.162 \times 10^{-4}$

Model from BE: $\varepsilon_{\mathrm{c}}=\frac{\Delta l}{l}=\frac{0.0218}{235}=0.928 \times 10^{-4}$

Comparison of numerical values of stresses and displacements obtained by finite element analysis of the investigated constructions with the corresponding values of these quantities that are obtained in the calculation by the boundary element method allows making a conclusion about the reliability of assumptions that were adopted at the stages of numerical simulation. This conclusion, perhaps, will be true with regard to the boundary conditions as well as the magnitude and nature of the application of external loads.

The obtained research results can serve as the basis for the development of new constructions for the treatment of diaphyseal fractures of the forearm.

\section{CONCLUSIONS}

The analysis of treatment methods of the forearm bones fractures is given. It is noted that existing devices are imperfect and therefore it is necessary to use modern and powerful numerical mechanics methods of a deformable solid to analyze the stress - strain state of biomechanical systems in order to improve them.

The analysis of numerical methods that are currently applied is performed. It is indicated that the most promising methods to solve the treatment problems and to improve orthopedic constructions are the finite element method and the numerical-analytical method of boundary elements.

The process of modeling the biomechanical system in the ANSYS professional package is shown in detail. Computed models of one-dimensional and spatial finite elements are compiled. The calculations of the SSS biomechanical system.

For applying BEM solutions of the Cauchy problems of tension - compression, shear, torsion, and bending were involved. A formation algorithm of a resolving system of equations is outlined. Appropriate calculations are made and compare son with FEM results is given where their close match is noted and thus the reliability of the results of both methods is proved.

A comparison of the numerical quantities of stresses and displacements obtained in the finite element analysis of the constructions with the corresponding quantities of these quantities obtained in the calculation using the boundary element method allows us to make a conclusion about the reliability of assumptions that were taken at the at the modeling stages. Perhaps, this conclusion will be true with regard to the boundary conditions, as well as the quantities and nature of the application of external loads.

\section{REFERENSES}

[1] De Backer, H., Outtier, A., \& Bogaert, P. (2014). Buckling design of steel tied-arch bridges. Journal of Constructional Steel Research, 103, 159-167. https://doi.org/10.1016/j.jcsr.2014.09.004

[2] Nilsen, C. L., Mydin, M. A. O., \& Ramli, M. (2012). Performance of lightweight thin-walled steel sections: theoretical and mathematical considerations. Advances in Applied Science Research, 3(5), 2847-2859.

[3] Pi, Y.-L. \& Bradford, M. A. (2013). In-plane stability of preloaded shallow arches against dynamic snap-through accounting for rotational end restraints. Engineering Structures, 56, 1496-1510. https://doi.org/10.1016/j.engstruct.2013.07.020

[4] Becque, J., Lecce, M., \& Rasmussen, K. J. R. (2008). The direct strength method for stainless steel compression members. Journal of Constructional Steel Research, 64(11), 1231-1238. https://doi.org/10.1016/j.jcsr.2008.07.007

[5] Andreev, V. I., Chepurenko, A. S., \& Yazyev, B. M. (2014). Energy Method in the Calculation Stability of Compressed Polymer Rods Considering Creep. Advanced Materials Research, 1004-1005, 257-260. https://doi.org/10.4028/www.scientific.net/amr.1004-1005.257

[6] Artyukhin, Yu. P. (2012). Approximate analytical method for studying deformations of spatial curvilinear bars. Uchenye zapiski Kazanskogo Universiteta. Physics and mathematics, 154, 97-111.

[7] Qiu, W.-L., Kao, C.-S., Kou, C.-H., Tsai, J.-L., \& Yang, G. (2010). Stability Analysis of Special-Shape Arch Bridge. Tamkang Journal of Science and Engineering, 13(4), 365-373.

[8] Pettit, J. R., Walker, A. E., \& Lowe, M. J. S. (2015). Improved Detection of Rough Defects for Ultrasonic Nondestructive Evaluation Inspections Based on Finite Element Modelling of Elastic Wave Scattering. IEEE Transactions on ultrasonics, ferroelectrics, and freguency control, 62(10), 1797-1808.

[9] Langer U., Schanz M., Steinbach O., \& Wendland W. L. (2012). Fast Boundary Element Methods in Engineering and Industrial Applications, Springer, 192-198.

[10] Orobey, V., Kolomiets, L., \& Lymarenko, A. (2015). Boundary element method in problem of plate elements bending of engineering structures. Metallurgical and Mining Industry, 4, 295-302. 
[11] Kolomiets, L., Orobey, V., \& Lymarenko, A. (2016). Method of boundary element in problems of stability of plane bending beams of rectangular cross section. Structures. Metallurgical and Mining Industry, 3, 59- 65.

[12] Orobey, V., Daschenko, O., Kolomiets, L., Lymarenko, O., \& Ovcharov, Y. (2017). Mathematical modeling of the stresseddeformed state of circular arches of specialized cranes. Eastern European Journal of Enterprise Technologies, 5/8(89), 4-11.

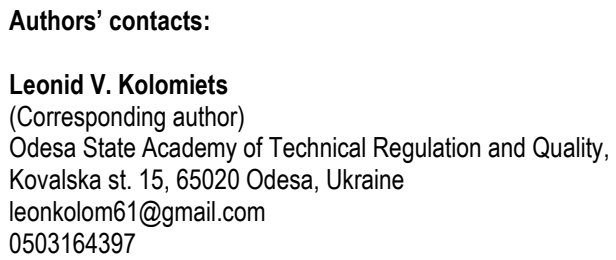

Aleksej Aniskin

University North, Department of Civil Engineering,

Jurja Križanića 31b, 42000 Varaždin, Croatia

aaniskin@unin.hr

Viktor F. Orobey

Odesa National Polytechnic University,

Shevchenko Avenue 1, 65044 Odesa, Ukraine

orobey51@gmail.com

0675570685

\section{Alexander Daschenko}

Odesa National Polytechnic University,

Shevchenko Avenue 1, 65044 Odesa, Ukraine

daschenko.o.f@opu.ua

0503329999.

\section{Aleksandr M. Lymarenko}

Odesa National Polytechnic University,

Shevchenko Avenue 1, 65044 Odesa, Ukraine

amlim@ukr.net

0675582617

\section{Kostiantyn F. Boriak}

Odesa State Academy of Technical Regulation and Quality,

Kovalska st. 15, 65020 Odesa, Ukraine

kostya.boryak@gmail.com

0661748263 